Article

\title{
A Circuity Temporal Analysis of Urban Street Networks Using Open Data: A Lisbon Case Study
}

\author{
Miguel Costa ${ }^{1,2}\left(\mathbb{D}\right.$, Manuel Marques $^{2}(\mathbb{D})$ and Filipe Moura ${ }^{1, *(\mathbb{C}}$ \\ 1 CERIS-Civil Engineering Research and Innovation for Sustainability, Instituto Superior Técnico, \\ Universidade de Lisboa, Av. Rovisco Pais 1, 1049-001 Lisboa, Portugal; mncosta@isr.tecnico.ulisboa.pt \\ 2 Institute for Systems and Robotics, Instituto Superior Técnico, Universidade de Lisboa, Av. Rovisco Pais 1, \\ 1049-001 Lisboa, Portugal; manuel@isr.tecnico.ulisboa.pt \\ * Correspondence: fmoura@tecnico.ulisboa.pt
}

Citation: Costa, M.; Marques, M.;

Moura, F. A Circuity Temporal

Analysis of Urban Street Networks Using Open Data: A Lisbon Case Study. ISPRS Int. J. Geo-Inf. 2021, 10, 453. https://doi.org/10.3390/ ijgi10070453

Academic Editors: Wolfgang Kainz and Giuseppe Borruso

Received: 29 April 2021

Accepted: 21 June 2021

Published: 1 July 2021

Publisher's Note: MDPI stays neutral with regard to jurisdictional claims in published maps and institutional affiliations.

Copyright: () 2021 by the authors. Licensee MDPI, Basel, Switzerland. This article is an open access article distributed under the terms and conditions of the Creative Commons Attribution (CC BY) license (https:// creativecommons.org/licenses/by/ $4.0 /)$.

\begin{abstract}
Urban street networks impact urban space usage and movement across a city. Circuity, the ratio of network distances to straight-line distances, is considered a critical measurement in urban network morphology and transportation efficiency as it can measure the attractiveness of routes in terms of distance traveled. Here, we compare circuity measures for drivable, cyclable, and walkable networks to analyze how they evolved and understand whether urban changes have produced meaningful circuity changes. Our analyses rely on Lisbon data from OpenStreetMaps to explore circuity for the period 2013-2020, which we used to simulate 4.8 million routes using OpenRouteService to compute the different modes' circuity measures. Our findings suggest that it is crucial to analyze each transport network type separately when planning or modeling urban street networks. Their composition and design differ significantly from mode to mode, such as their attractiveness to users. We identify significant changes in modes' circuity over time, especially in cycling, following Lisbon's cycling infrastructure expansion. Our paper demonstrates that the circuity indicator is useful when planning and modeling street networks, in particular, to optimize the location choice for interventions required to increase the attractiveness of active modes and promote sustainable mobility. At the same time, we emphasize the lack of information on walking infrastructures required for more detailed analyses.
\end{abstract}

Keywords: circuity; urban street networks; cycling infrastructure changes; accessibility; Lisbon (Portugal)

\section{Introduction}

Urban streets shape how people use space for both accessibility and mobility across a city. They facilitate or deter socioeconomic activity and environmental sustainability, such as job locations, commuting trips, and modal share. Therefore, the urban street's intrinsic design influences how cities grow and their transportation needs over time [1]. For instance, past urban planning policies optimized urban streets for motorized traffic while ill-treating pedestrians and cyclists by imposing constraints and exposing them to dangers. Conversely, current policies are now trying to move in a more sustainable direction by targeting transportation systems towards more efficient and sustainable solutions, focusing on providing safe, comfortable, and positive experiences for pedestrians and cyclists, since these users place a relatively high value on certain route characteristics [2,3]

The built environment has a notable effect on travel decisions, and so spatial context is vital [2]. Analyzing the relationship between physical space, urban form, accessibility, and transportation becomes key to understanding how transportation systems can be adapted and improved to benefit everyone. Identifying how city areas and their street networks serve distinct transportation modes and how unbiased these networks are, is becoming key to providing spatial equity for the entire population. The use of the Gini coefficient and Lorenz curves can help drive policy towards such spatial inequity [4]. Furthermore, studying travel mode choice and how routes can promote or deter the choice of a given mode becomes even 
more important when cities seek to promote more sustainable travel modes, such as cycling and walking.

Analyzing how urban streets perform, both in terms of efficiency (to meet some functioning target) and use (to satisfy demand) can help understand how to promote such positive experiences. We base our work on this assumption by using circuity (a measure of urban network efficiency [5]), together with open data and tools, ultimately fostering the use of an easily repeatable methodology to analyze how streets are different for different transportation modes. Circuity (also called detour index or route factor [6]) measures network distances' ratio to straight-line distances. In short, circuity serves as a basis for measuring the attractiveness of routes, and it can serve as a starting point in the comparison of city areas, districts, or even whole cities from a network performance perspective. In our work, we measure and compare the circuity of three distinct networks: drivable, cyclable, and walkable street networks.

The thought of describing and analyzing how street networks impact cities' liveability and enjoyment is not new [7,8]. Urban network analysis primarily arises from graph theory and how network topology can describe both structure and efficiency [9]. Network characteristics and respective metrics, such as connectivity, density, and centrality, help to differentiate network designs and, therefore, expectable travel intensities [8,10]. The growing interest in understanding urban network structure has led to research on urban metrics such as completeness [8], connectivity [10], treeness [10], centrality [11], street density [8], street bearings, and street orientation entropy [12], among other things. Ultimately, by trying to quantify cities' spatial structure of transportation networks, these metrics can support urban planning as they are tools to simulate different scenarios and compare different network systems $[7,13]$. In turn, this may enable planners and decision-makers to make faster and more accurate decisions on areas that may need intervention $[9,11,14]$.

Quantitative measures of the underlying network explore different transportation system's performance (supply side), together with travel data (demand side) [8]. Here, Reference [8] notes that greater connectivity leads to less travel, and thus, quantitative measures should be considered when designing and planning new infrastructures. Similarly, ref. [14] suggests how network indicators can evaluate transportation performance. In this case, average closeness centrality and road connectivity over average road circuity identify situations where one could ease road congestion.

In this work, although planning should analyze and combine different metrics, we focus only on circuity as it is a direct result of how networks are planned and built [12], and, in comparison, street network configurations impact how people use street networks for both their accessibility (to reach the desired activity locations) and mobility (usageintensity of a segment) $[15,16]$. Circuity, i.e., the traveled distance and straight-line distance ratio, can measure locations' attractiveness because trips with lower circuity trips imply fewer detours and become more attractive in the distance traveled. Research has analyzed the relation between home locations and workplaces based on lower circuity values [16] or exploring how circuity helps explaining transit accessibility to jobs and how it can significantly assist in explaining commuting mode share across different U.S. metropolitan cities [5]. Similarly, Cubukcu [6] uses circuity to examine the network efficiency of Paris. As noted, circuity varies with distance traveled, and it is not homogeneous across space, suggesting the hypothesis that different city districts have to be analyzed individually to evaluate its efficiency better.

Circuity has been studied before for different transportation modes: car [6,17-19], bicycle [19], walking [18-21], and transit [5]. Different transportation modes use different types of networks. For example, pedestrians may use sidewalks, whereas cars cannot, and vice versa, cars may use freeways while pedestrians cannot. Analyzing each transport network's circuity separately is essential as each distinct transportation mode can be associated with a different network. Comparisons between different modes have also been studied in several U.S. cities [18] and Thailand's capital [19], and statistical differences were found between driving, walking, and cycling. Results show that walking trips' circuity 
is typically lower than cycling or driving. Nevertheless, Yen et al. [19] and Cubukcu [6] also note a distinction on how circuity measures vary from city district to city district, suggesting that we should analyze circuity at a city district level for a better comprehension of the network. However, circuity has not been evaluated longitudinally across time.

This paper's main objectives are threefold:

1. To present a methodology based on open data and tools for analyzing different transport modes' circuity in urban street networks;

2. To compare and analyze circuity based on two distinct sampling strategies that serve to understand urban street networks under two scenarios: isotropy (analyze the network as a whole) or anisotropy (analyze the network that people use); and

3. To analyze the evolution of circuity across time and explore if (and how) infrastructure changes have influenced circuity in urban networks.

We use Lisbon, Portugal as a case study and analyze circuity for three urban street networks: drivable, walkable, and cyclable circulation. Lisbon has changed in recent years, with the municipality investing more in both cycling and walking infrastructures. We use the presented methodology to understand whether municipalities' interventions have produced meaningful circuity changes for all users over time and whether these changes have resulted in better circuity for the network as a whole, or to the network that people actually use. We base our procedure on open data from OpenStreetMaps (OSM) to explore circuity for 2013-2020, easily replicable in any other region (mapped by OSM). These data include drivable, walkable, and cyclable networks, which we then use to simulate 4.8 million routes using OpenRouteService. Next, we compute circuity measures longitudinally across time (yearly analysis) and for the different modes. To the best of our knowledge, this isotropic vs. anisotropic and temporal analysis has not been performed in the past.

We organize the paper as follows: after this introductory section, we present the data, materials, and methodology used in Section 2, including a brief description of Lisbon as our case study. Next, in Section 3, results from our circuity calculations are enumerated and discussed further in the Discussion section. Finally, conclusions finalize the paper.

\section{Materials and Methods}

Here, we compared circuity measures for Lisbon, Portugal, across three distinct urban street networks: drivable, cyclable, and walkable networks. In short, using the networks for each mode, we started by selecting origin and destination points. Next, we computed the routes for each origin and destination (OD) pair and each modal network. Then, knowing the network distance (from the previously computed routes) and straight-line distance for each OD trip, we computed the respective circuity for all three network types. We end by giving an overview of our case study: Lisbon, Portugal. All the code and materials used are available in https:/ / github.com/U-Shift/ circuity-lisbon, accessed on 28 June 2021.

\subsection{Data and Networks}

First, we extracted the network file corresponding to our case study. In our case, we began by extracting the entire OpenStreetMap history file for Portugal from GeoFabrik (https:/ / download.geofabrik.de/ europe / portugal.html, accessed on 11 June 2020). OpenStreetMap data consist of geographic information of the world created by users who voluntarily contribute to its creation, edition, and expansion. Its data represent physical characteristics (e.g., roads, trees, buildings, or traffic signals) and their location in the world. Each element can be detailed (e.g., type of road, number of lanes), enabling different network' topologies to be defined. Here, we used this information and network differences to compute routes using different transport modes. Different modes have different requirements about what type of infrastructure they can use (e.g., bicycles cannot cycle in motorways), and hence different routes can be computed to reflect modes' routing options. OpenStreeMap history files contain the complete OpenStreetMaps data over time, with timestamps associated with every element from when it was created, edited, 
or deleted. This allows inspecting the network state at any given point in time, allowing researchers to inspect the state of a given network at any time period.

Next, we filtered Lisbon's municipality limits and drew the convex hull over these limits from the downloaded history file. We filtered using this convex hull such that more short and direct routes were considered, even if they cross Lisbon's municipality borders [18]. Next, we extracted Lisbon's data at different time periods by filtering Lisbon's history file accordingly. We sampled yearly map snapshots from 2013 to 2020 (we extract the network state on the 1st of January of each year), totaling eight different networks, one for each year. More fine filtering could be performed to achieve greater accuracy in network changes over time, but we considered this yearly interval to be short enough to capture significant urban changes and long enough not to capture any creation or editing errors that users may input into OpenStreetMap. Additionally, since OSM data are collaborative, we checked the quality of the data. Cycling infrastructures have been manually compared to authoritative data from Lisbon's municipality regarding cycling lanes and paths. No data were available from official sources regarding the road network to perform any comparison to OSM data. An analysis of the OSM data has shown that there has been a change of only $6 \%$ in length for the 2013-2020 period, denoting mapping maturity. Additionally, Ref. [22] estimated that on 1 January 2016, Portugal's road data was complete, but about 0.8 complete in 2013. Lisbon, being the capital and most developed urban region in the country, is expected to be much more complete than other, more remote, areas in Portugal. Finally, regarding walking infrastructure, there are no authoritative data from the municipality and so no comparison can be made with the data from OSM.

\subsection{Origin and Destination Sampling}

Secondly, we sampled origin and destination points across Lisbon. We sampled 100,000 pairs of OD points to ensure that the entire city is covered. Later, each OD pair was to be used to simulate a walkable, cyclable, and drivable route. We chose to sample 100,000 pairs of points to ensure that a sufficient number of OD pairs covers the entire city. We evaluated the city's networks for two scenarios: (i) isotropic network, assuming transport demand uniformity over the city, and (ii) anisotropic network, where transport demand varies over the city. Accordingly, we used two sampling strategies: Random Sampling (RS) and Mobility Survey Sampling (MSS). This procedure yielded two different sets of 100,000 OD pairs, where each set originates from one of the sampling techniques. Random Sampling (RS) focuses on equally distributing origin and destination points throughout the city. This sampling technique ensures isotropy and that all city areas have equal probability of being chosen and is more appropriate for analyzing the entire city network. Mobility Survey Sampling (MSS), on the other hand, samples points based on observed trips and their OD pairs. A mobility survey (IMob-Inquérito à Mobilidade) on Lisbon's metropolitan area was conducted in 2017 to understand the mobility of the population, its daily patterns, reasons, and opinions about each available transport mode. We collected an anonymous sample of trips from this survey, including the origin and destination points for each trip and respective transport modes. As IMob does not contain users' routes, we sampled its OD points following its trip OD distribution. This sampling procedure ensures anisotropy and that the OD distribution follows observed individuals trips, more suitable for analyzing the street network that people use.

\subsection{Route Simulation}

Thirdly, we performed route simulation for the three modes (walking, cycling, and driving) using OpenRouteService (https: / / openrouteservice.org/, accessed on 28 June 2021). We installed a private server of OpenRouteService (https: / / github.com/GIScience/openr outeservice, accessed on 28 June 2021) and made use of its Directions service to query routes between each OD pair for each sampling technique. This service acts as a trip planner and returns a route between two locations for any given mode using the urban street network provided. Thus, we simulated routes for each OD pair (100,000 pairs of points) with each 
sampling technique (2 sampling techniques) using the OpenStreetMap network from each year (8 years for the period 2013-2020), for each of the selected transport modes (walking, cycling, and driving), totaling 4.8 million simulated routes.

To simulate all three modes, we used OpenRouteService default profiles: driving-car (driving), cycling-regular (cycling), and foot-walking (walking). Profiles can be set up such that different restrictions are made for different modes, enabling the customization of routes depending on network attributes, such as road types, speed limits, grades, and others. We used OpenRouteService's pre-defined profiles because of their simplicity to use and suitability for the task. The fastest route was simulated for each mode. Details about how each profile routes trips can be found elsewhere [23]. In short, driving trips are routed through the fastest route with roads where motorized vehicles can traverse. Cycling trips are routed such that trips cannot pass through certain road types (e.g., motorways), and a preference is given for routes that pass through cycleways when possible, which relates to ongoing research about cyclists' preferences about route choices $[3,24,25]$. Finally, walking trips are routed preferably through sidewalks, footways, or other paths that allow pedestrians to move. In sum, we simulated the fastest route for each mode, totaling three different routes for each OD pair. Figure 1 shows the differences for the three modes for a single OD pair.

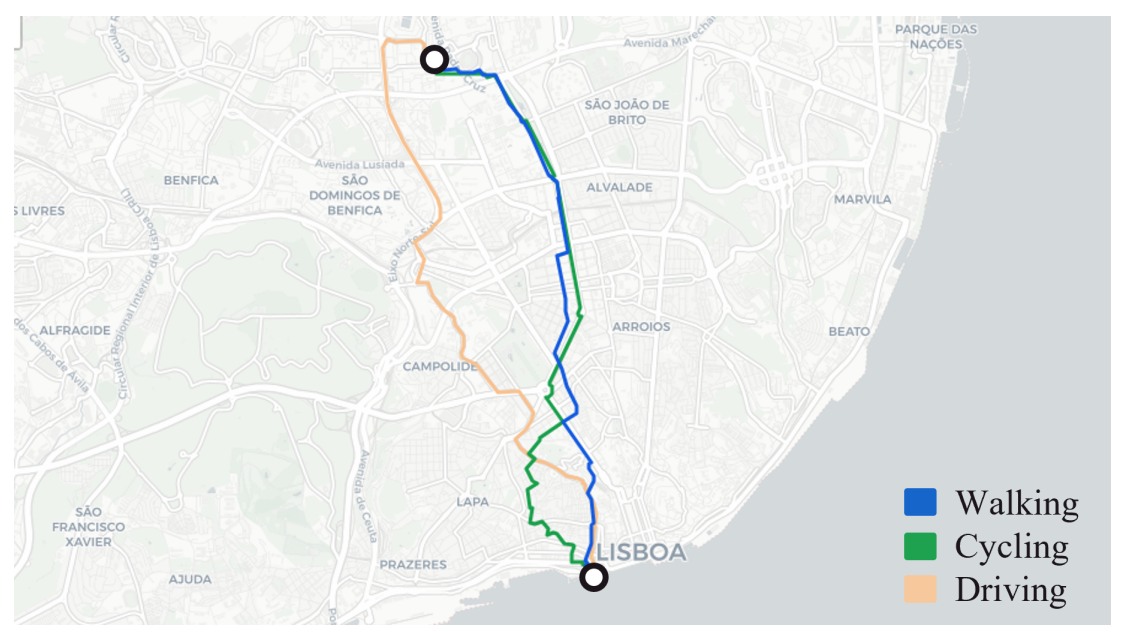

(a)

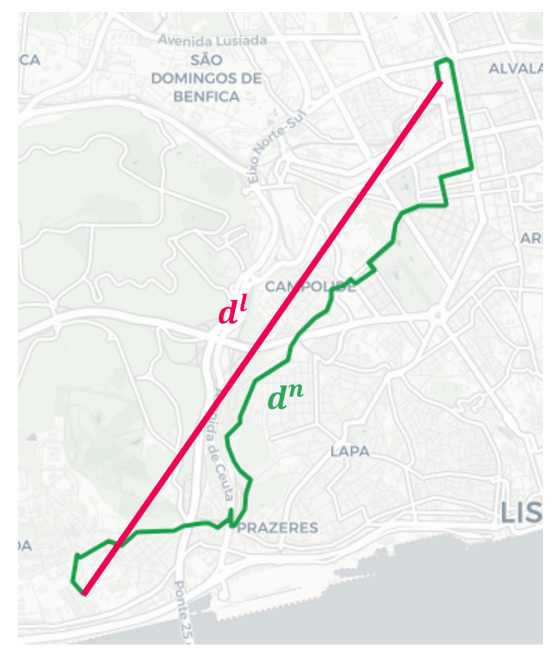

(b)

Figure 1. Driving (orange), cycling (green), and walking (blue) routes for an Origin-Destination pair (a), and circuity as the ratio of network distances and straight-line distance of one trip (b).

\subsection{Circuity}

Circuity measures the ratio of network distances to straight-line distances. Figure 1 exemplifies these two distances for a single trip. It can be defined as:

$$
C_{i k}=\frac{d_{i k}^{n}}{d_{i}^{l}},
$$

where $C_{i k}$ is the value of circuity between network distance $d_{i k}^{n}$ and straight-line distance $d_{i k^{\prime}}^{l}$ for a pair of origin-destination points $i$ and mode $k$. The straight-line distance corresponds to the greater-circle distance between two points (origin and destination) and can be computed using:

$$
d_{i k}^{l}=r \arccos \left(\sin \alpha_{O} \sin \alpha_{D}+\cos \alpha_{O} \cos \alpha_{D} \cos \left|\beta_{O}-\beta_{D}\right|\right),
$$

where $r$ represents the Earth's radius and $\alpha_{O}, \beta_{O}$ and $\alpha_{D}, \beta_{D}$ correspond to the origin and destination points and latitude and longitude in radians, respectively. This distance matches the shortest distance between the two points along Earth's curvature surface and 
is more rigorous than the Euclidean distance. The network distance $d_{i k}^{n}$ is the distance of the computed route using OpenRouteService between OD pair $i$ for mode $k \in\{$ driving, cycling, walking\}.

\subsection{Our Case Study: Lisbon}

Spanning over $100 \mathrm{~km}^{2}$, about half a million people reside in Lisbon (nearly $5 \%$ of Portugal's population). Lisbon comprises 24 parishes with different areas, populations, and residential or workplace densities. The parishes of Santa Maria Maior, Misericórdia, São Vicente, and Santo António correspond to the historical city center and downtown, where many touristic attractions are located. Avenidas Novas and Parque das Nações are Lisbon's Central Business Districts, where major multinational and national companies are located. Other parishes, such as Lumiar and Benfica, correspond to residential districts. Figure 2 shows the location of each parish in Lisbon. Lisbon's parishes are very diverse regarding access to transport networks, whether for transit users, cyclists, or pedestrians.

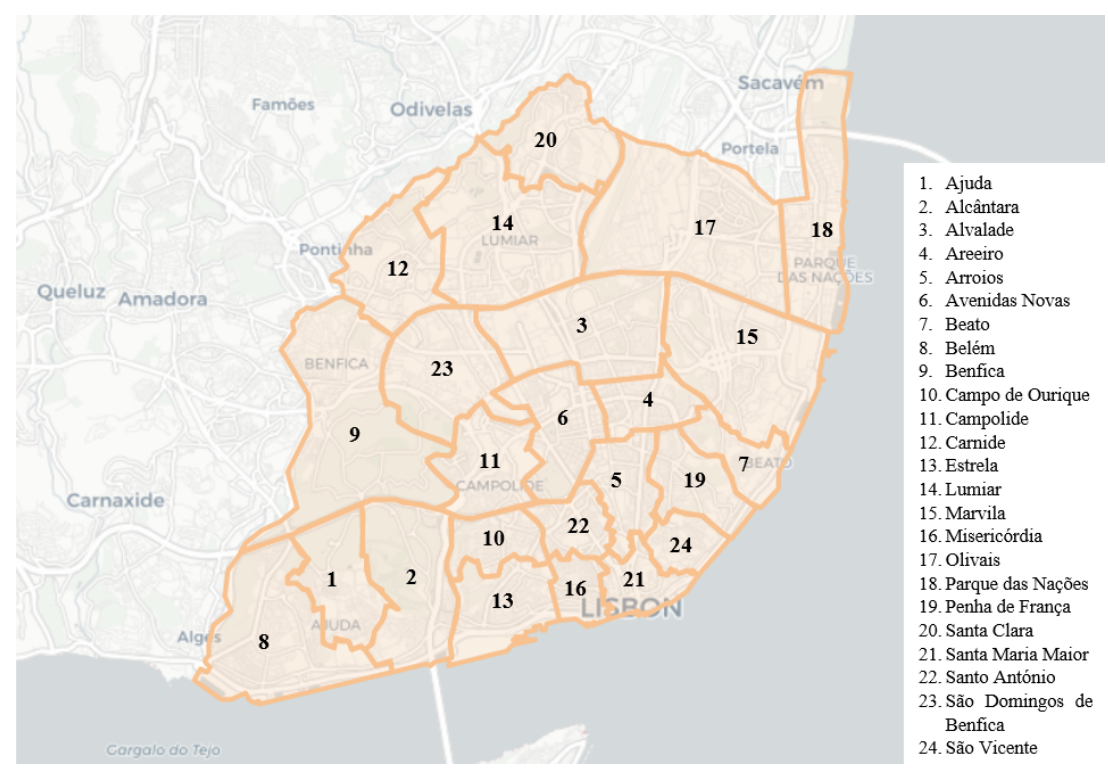

Figure 2. Lisbon's parishes.

The latest survey of Lisbonians' mobility was conducted in 2017 to characterize their trips' origins and destinations, travel times, distances, and costs. IMob ("Inquérito à Mobilidade"-Mobility Survey) showed that Lisbon's residents each day make a total of 935.253 trips. Trips average a travel time of $23.1 \mathrm{~min}$ and a trip distance of $4.2 \mathrm{~km}$ [26]. Private motorized vehicles were the most used with $46 \%$ modal share, public transportation accounted for $22 \%$ of all travels, $29.8 \%$ walked, and only $0.6 \%$ cycled [26]. Despite being perceived as a hilly city, more than half $(54 \%)$ of the streets are almost flat $(<3 \%$ steepness) [27]. Short average distances, combined with low gradient streets, allow for a greater number of walking trips. Cycling numbers remain relatively low as a highly disconnected cycling network, lack of places to store or lock bikes safely, and a general perception of an unsafe cycling environment are significant barriers to start cycling regularly [28].

Following the 2008 Recession, Portugal has adopted tourism and urban rehabilitation to overcome the profound financial crisis [29]. Lisbon has been no different, and many changes have occurred throughout the city. The historical city center has suffered many transformations. Some of the $18 \mathrm{~km}$ long riverbank have been rehabilitated to improve pedestrian accessibility, and programs such as "Uma Praça em Cada Bairro" (A Square for Every Neighborhood) have increased accessibility to local commerce and jobs. These changes also promote public transportation and active modes while deterring car usage by imposing parking or access time constraints. The Lisbon municipality has also revamped Lisbon's Central Business District (CBD) since 2016, transforming urban mobility by re- 
ducing arterial avenues' car capacity and speed limits, tackling pressing climate changes. Simultaneously, a more connected and commuting-oriented cycling network has been created, mainly in the CBD and the Tagus River waterfront. Figure 3 shows how the cycling network (with dedicated cycling lanes) has evolved in Lisbon in recent years. The cycling modal share from $0.2 \%$ in 2011 [30] to $0.6 \%$ in 2018 [26], and the expansion of cycling infrastructures and the implementation of a bike-sharing system have been vital to the growth of cycling maturity in Lisbon [31].
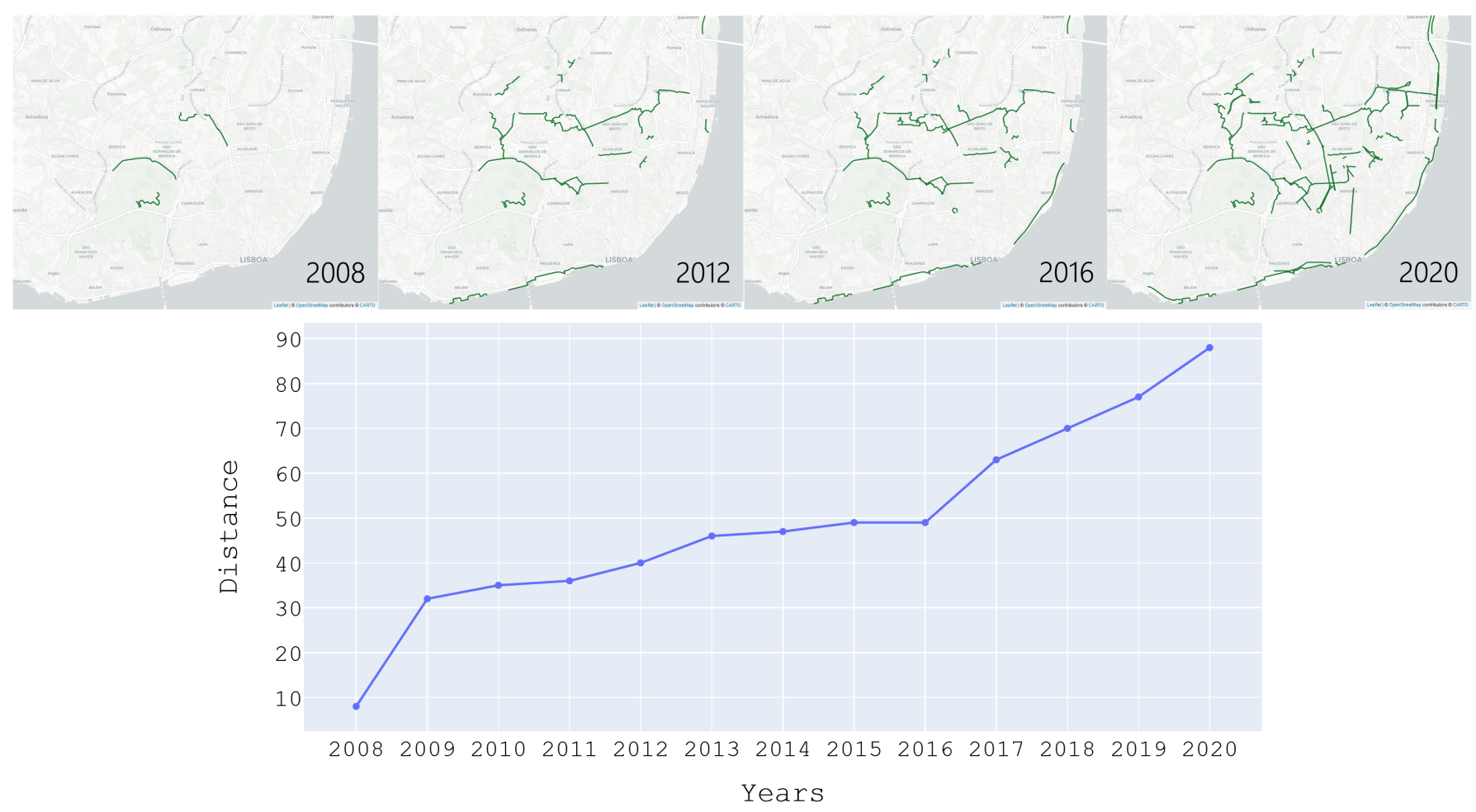

Figure 3. Evolution of Lisbon's cycling network between 2008 and 2020 and its total distance in km [32].

\section{Results}

Following the simulation of 100,000 trips for each sampling technique, we computed the circuity values for all three transport modes between 2013 and 2020. In total, we simulated 4.8 million routes and calculated the corresponding circuity. Next, we present a series of results that we found in our analysis. First, we show the evolution of the average circuity over time in Lisbon. We detail circuity for the three modes and point out how circuity differs for different traveled distances. Second, we show the main differences between the two sampling techniques: MSS and RS. Based on this analysis, we zoom in on our analyses at Lisbon's parishes scale. Noticeably, parishes' circuities are very distinct. More, we see how the cycling infrastructure expansion had a remarkable impact on cycling circuity values. Fourth, we take a more refined look at the types of roads (way types) used per trip mode. We could spot where each segment of each trip was made, allowing for a contextual inspection of trips. Finally, we use the Gini coefficient to diagnose any significant inequalities between drivable, cyclable, and walkable networks.

\subsection{Average Circuity}

Figure 4 shows the average circuity for driving, cycling, and walking trips for the two sampling techniques (MSS and RS) between 2013 and 2020. We notice a decreasing trend in circuity values for cycling and walking, whereas such a trend is not evident for driving trips. In particular, 2016 appears to be a turning point for both active modes, which seems to match Lisbon's greater expansion of the cycling network (cf. Figure 3). Furthermore, driving trips are more circuitous than cycling trips, which are more circuitous than walking 
trips. Finally, we see that RS trips exhibit lower levels of circuity for all modes when compared to MSS.

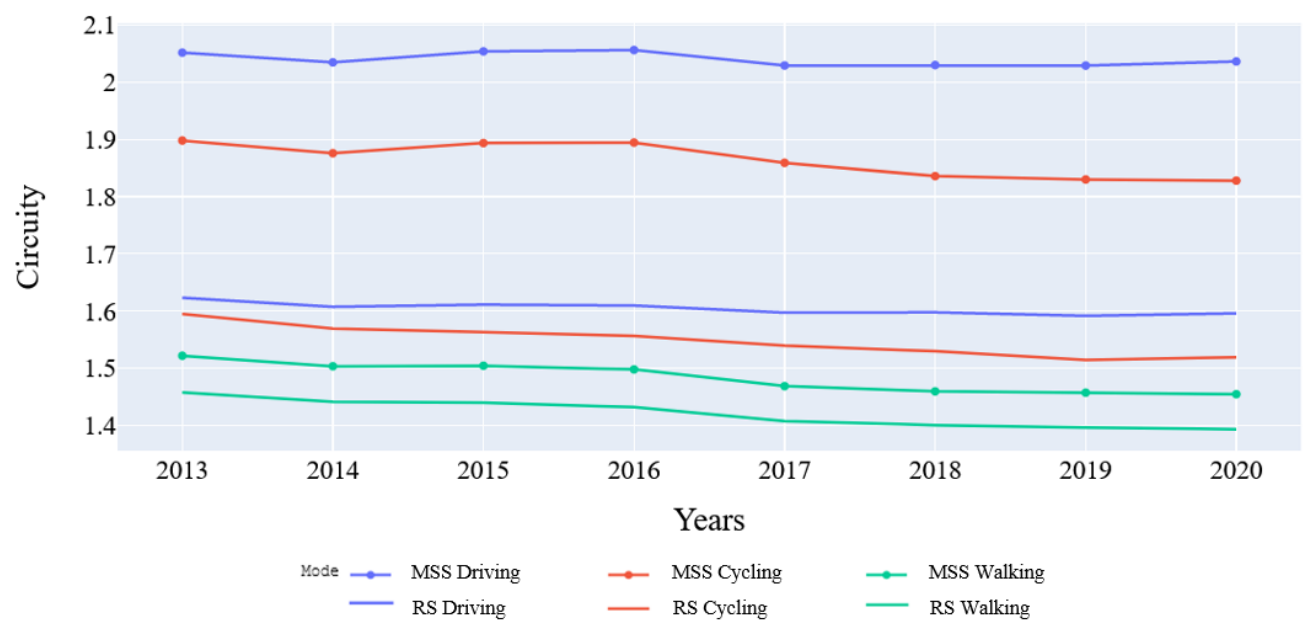

Figure 4. Average circuity for driving, cycling, and walking for 2013-2020 for the two sampling technique (MSS and RS).

While mean circuity helps in a generalized analysis, a more detailed inspection can be performed if trips are categorized according to the distance traveled. Figure 5 shows MSS and RS trips categorized across four distance intervals: 0-1 km, 1-2 km, 2-8 km, and 8-12 km. Again, circuity decreases as trip distance increases, with trips up to a kilometer being highly circuitous for cars and bicycles. Interestingly, for trips over a kilometer, RS trips become slightly more circuitous than MSS trips. Between 2 and 8 km, 2013-2014 car trips are as circuitous as bicycle trips. These became slightly less circuitous from 2015 onwards. For trips above $8 \mathrm{~km}$, cycling becomes more circuitous than driving, regardless of the sampling technique. Cycling trips prefer to use cycling infrastructure. For greater trip distances, cyclists will route to more circuitous routes, increasing circuity levels. This shows that there is a lack of connectivity between distant regions in Lisbon.

Finally, we check to see the variation of cycling average circuity with the changes in total cycling lanes length in Lisbon. From 2013 to 2020, 55.4 km of cycleways were built in Lisbon, in which there was a reduction in the average cycling circuity of about 0.09 . More noticeably, between 2016 and 2017, $23.15 \mathrm{~km}$ of cycleways were built, coinciding with the biggest decrease in circuity during the same period, as seen in Figure 4 (about $50 \%$ of the total decrease for the period 2013-2020).

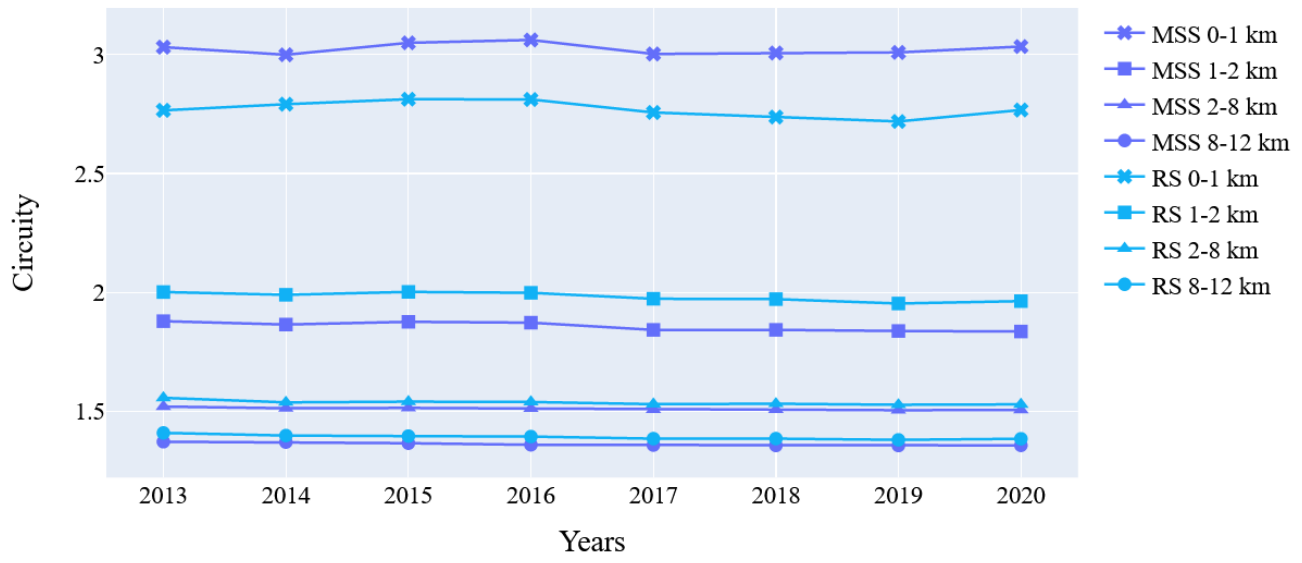

(a) Driving

Figure 5. Cont. 

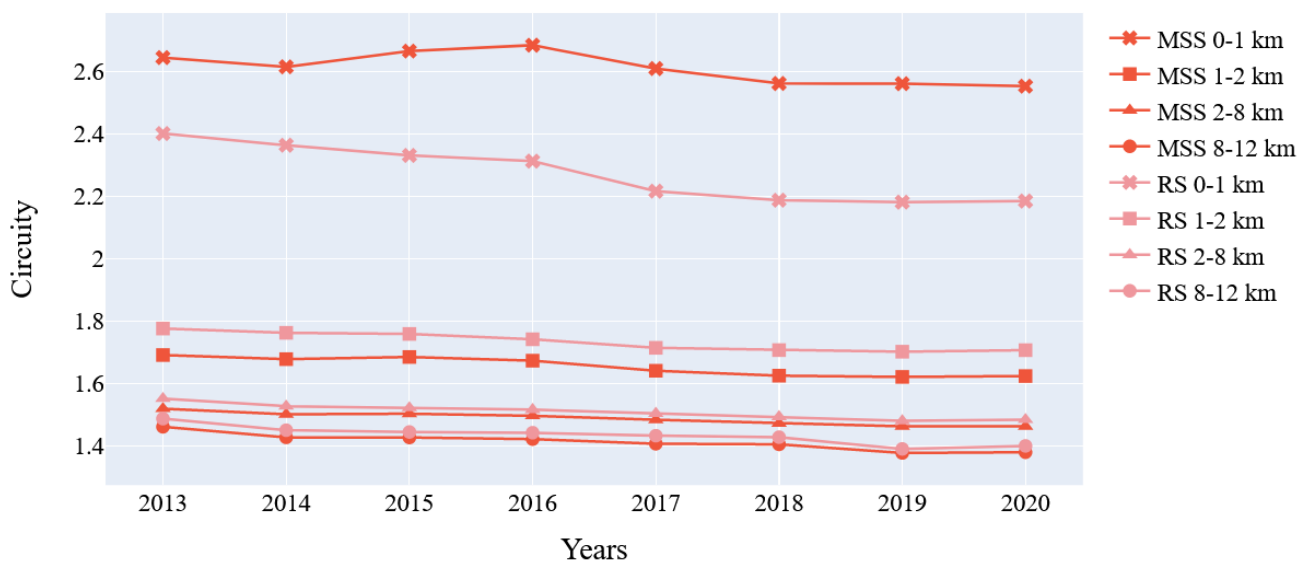

(b) Cycling

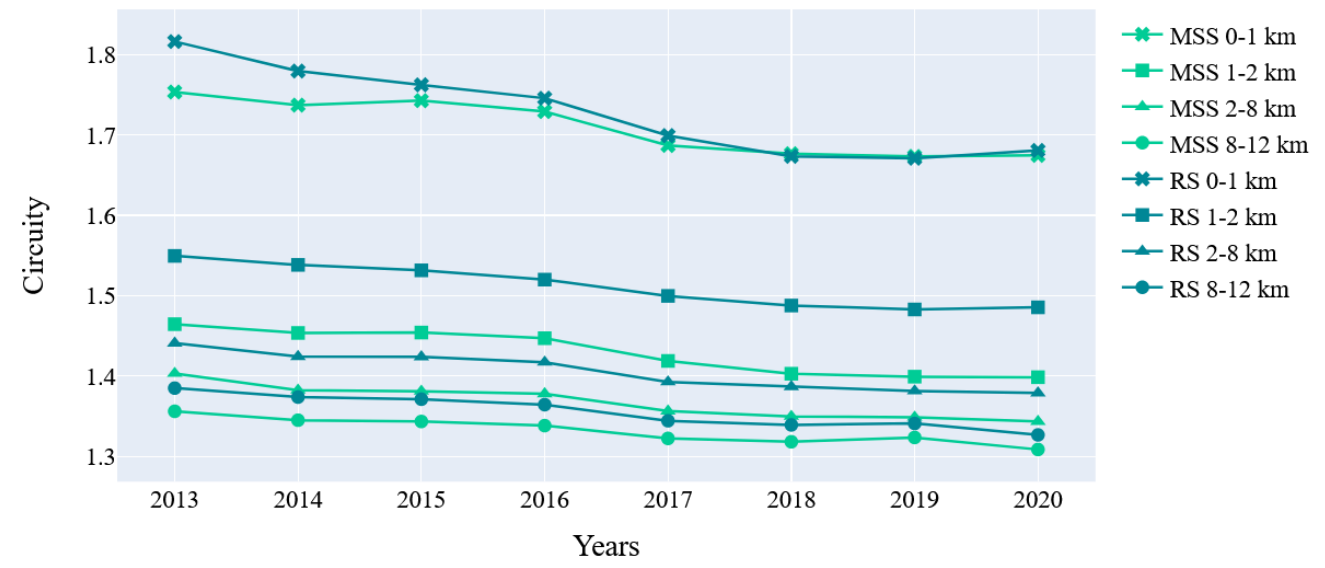

(c) Walking

Figure 5. Average circuity for driving, cycling and walking for the period 2013-2020 for the two sampling techniques (MSS and RS) for different trips' distances.

\subsection{MSS vs. RS}

Next, we compare the two sampling techniques: MSS and RS. Looking at the trips' starting points and destination points, we note that despite OD points being spread through the city, RS and MSS only share the same origin and destination parishes in $3 \%$ of all trips, meaning that OD pairs are almost non-overlapping. This difference highlights the chosen OD points between the two sampling strategies, highlighting that MSS shows preferential trips made by its citizens.

Figure 6 illustrates the density of trips' origins per Lisbon parishes' area. As we can see, there are significant differences between the two sampling techniques. For one, RS density is approximately equal for all parishes. However, this is not the case for MSS, where density is higher for parishes near the CBD and its surroundings, such as Avenidas Novas, Arroios, Misericórdia, and Santo António. Results are similar if we ought to consider density per destination point. This result highlights the differences between the two techniques. While RS assumes an isotropic territory and thus selecting points from the entire city evenly, MSS assumes an anisotropic territory and captures locations that generate or attract people and trips. Such results emphasize the hypothesis that both sampling techniques should be used for different purposes: RS should be used for an isotropic analysis of the entire territory (e.g., as a proxy of the full territory's potential mobility), and MSS ought to be used for analyzing citizen's mobility needs and, therefore, their trips. 


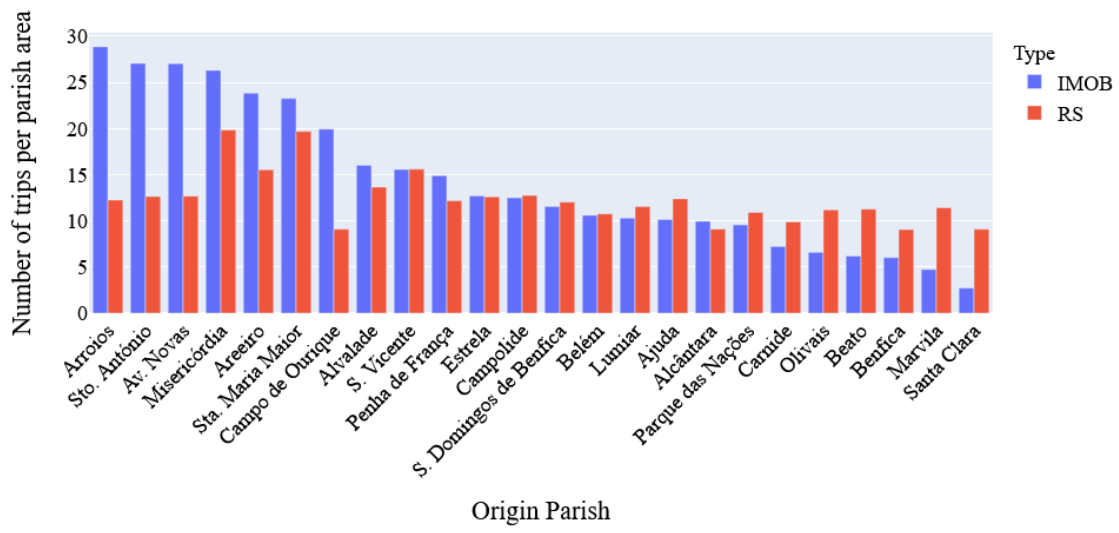

Figure 6. Trips' origin density per parish area $\left(\mathrm{km}^{2}\right)$ for both MSS and RS sampling.

\subsection{Lisbon's Parishes Circuities}

Figure 7 depicts the median circuity for trips that start and end on the same parish relative to different distance categories for the year 2020. We use 2020 as a case study and MSS as the sampling technique to better evaluate how the current network performs for intrazonal trips, i.e., trips that begin and end within the same parish. As we can see, there are significant differences in how driving, cycling, and walking perform for Lisbon's 24 parishes. In general, for shorter trips $(<2 \mathrm{~km})$, driving is more circuitous than cycling, which is more circuitous than walking. For trips between 2 and $8 \mathrm{~km}$, car trips are as circuitous as bicycle trips, except for the Ajuda parish. Lastly, for trips over $8 \mathrm{~km}$, the car seems the best option compared to cycling and even walking in some cases. Considering Lisbonians' average trip distance of $4.2 \mathrm{~km}$, we can note that for this distance category $(2-8 \mathrm{~km})$, the car seems as attractive as cycling when it comes to the distance traveled, which may help to explain Lisbon's car dominance but also suggests that there is a potential for cycling uptake.

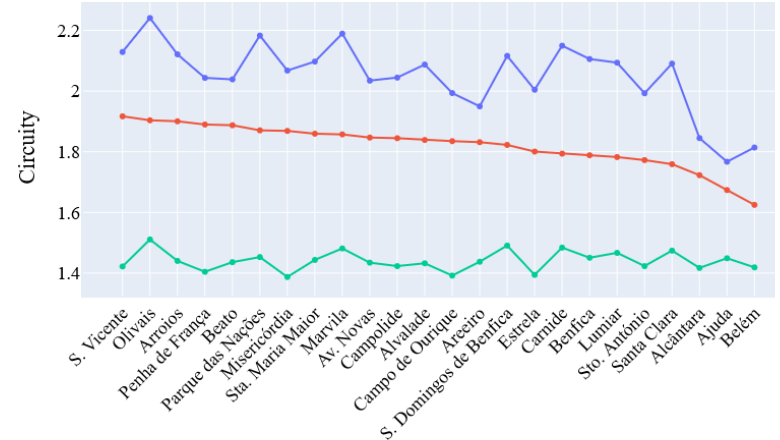

(a) Trips' distance $0-1 \mathrm{~km}$

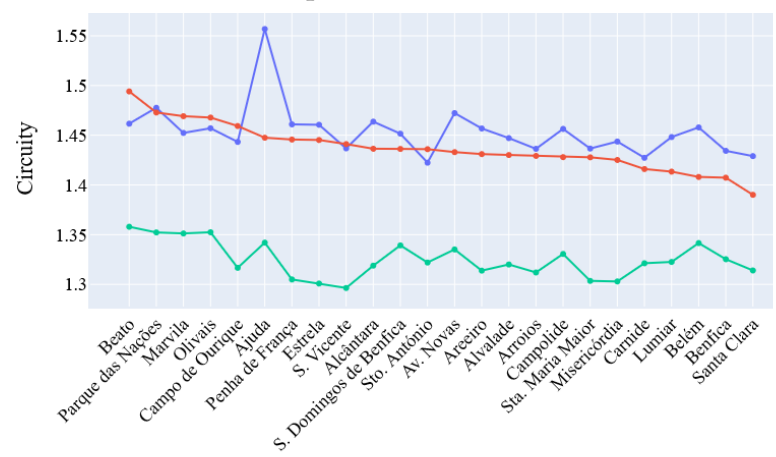

(c) Trips' distance $2-8 \mathrm{~km}$

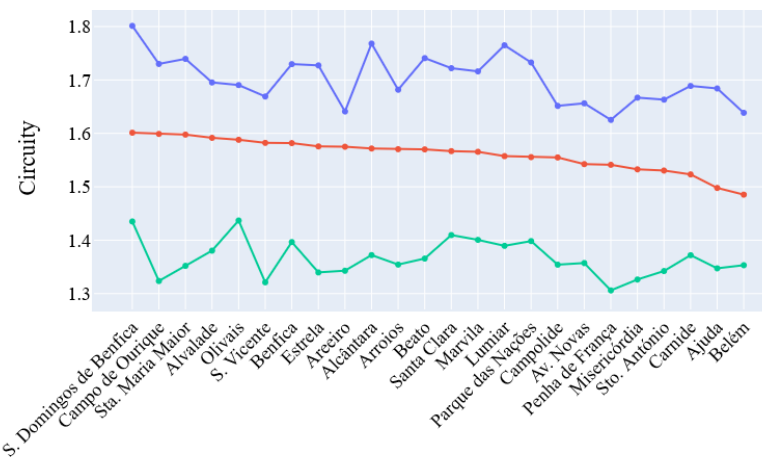

(b) Trips' distance $1-2 \mathrm{~km}$

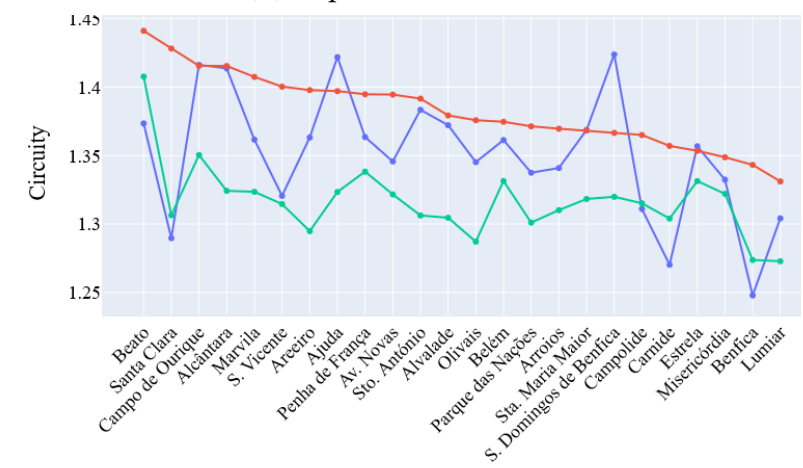

(d) Trips' distance $8-12 \mathrm{~km}$

$$
\text { Mode } \rightarrow \text { driving } \rightarrow \text { cycling } \rightarrow \text { walking }
$$

Figure 7. Median in-parish trips circuity for driving, cycling, and walking in 2020 using MSS. 
Instead, if we consider our analysis's entire period, we note some other particularities of how circuity has changed over time. Figures 8 and 9 illustrate the evolution of the median circuity between 2013 and 2020 for trips between 2 and $8 \mathrm{~km}$ using the MSS approach for two parishes in Lisbon: Marvila and Olivais. Both parishes had multiple infrastructure interventions with, amongst others, the construction of cycleways designed to link the CBD with these parishes better. In Marvila, cycling infrastructures increased near one of its core avenues and the riverbank from2016 to 2017, with a supplementary expansion in 2018. Since then, the network has remained unchanged. Similarly, between 2016 and 2018, we see a sharp decrease in cycling's circuity, coinciding with the infrastructure changes. The same happened in Olivais. We noted a decreasing circuity from 2015 to 2019 and stagnation in 2019-2020. The parish also suffered infrastructure changes up until 2020. However, it appears that the infrastructure changes in 2020 did not produce any meaningful circuity changes, disputing its efficiency.

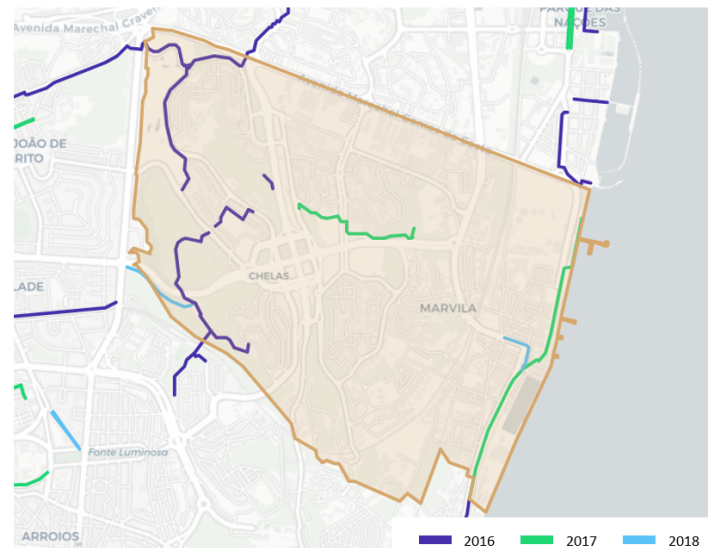

(a)

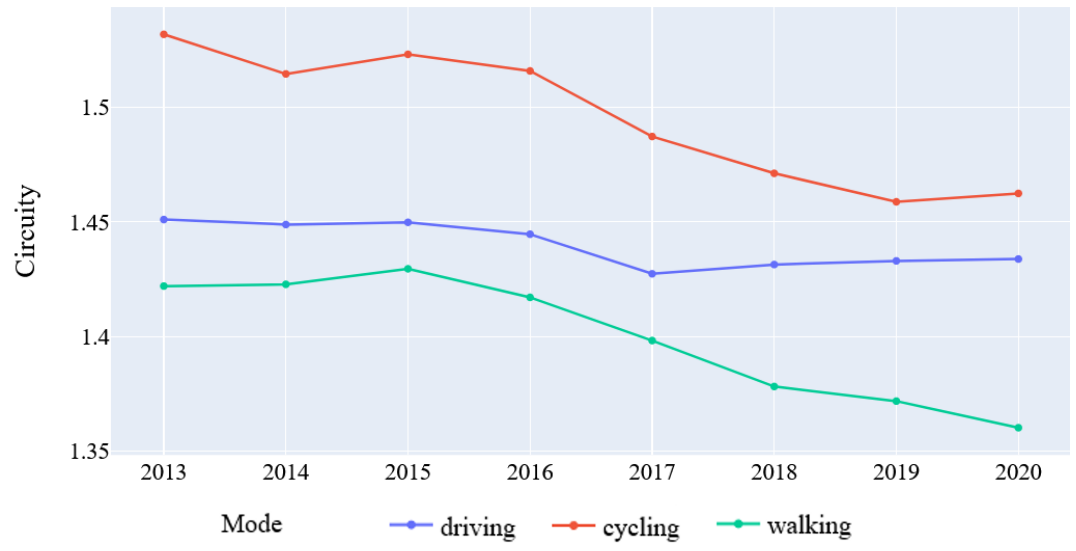

(b)

Figure 8. Cycling infrastructure changes from 2016 to 2018 in Lisbon's parish of Marvila (a), and the evolution of the median circuity in Marvila (b).

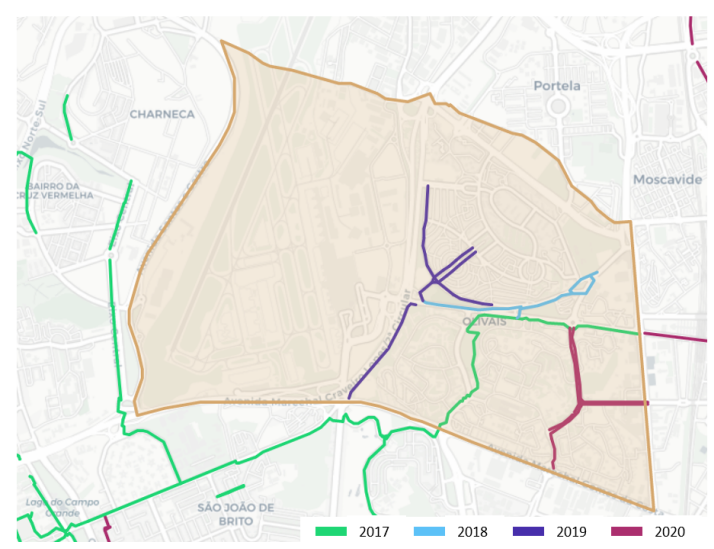

(a)

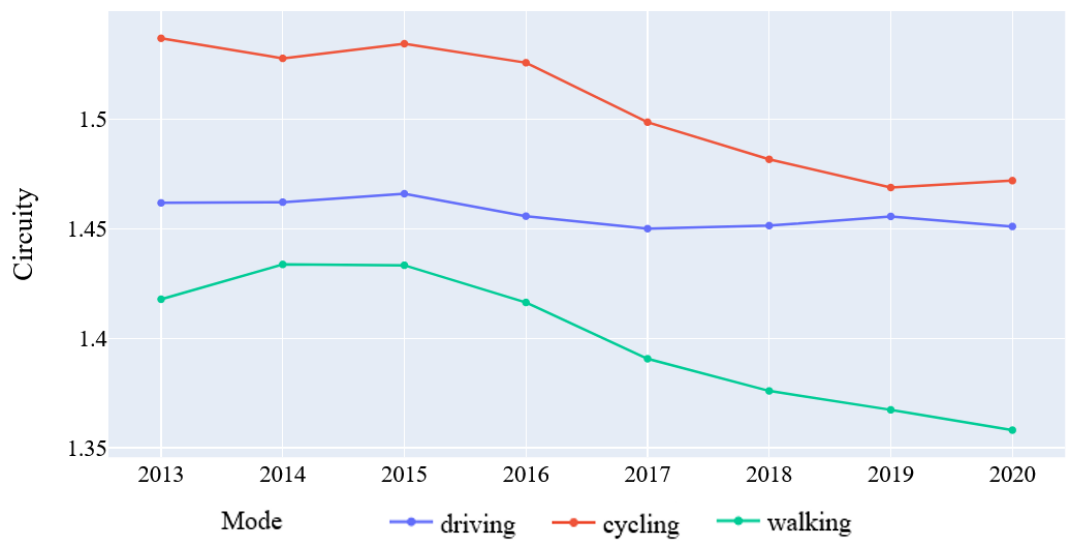

(b)

Figure 9. Cycling infrastructure changes from 2017 to 2020 in Lisbon's parish of Olivais (a), and the evolution of the median circuity in Olivais (b).

\subsection{Waytypes}

OpenRouteService classifies roads into several categories, including State Roads, Roads, Streets, Paths, Cycleways, Footways, and other less frequent types. Our subsequent analysis scrutinizes the typology of roads that each mode uses, particularly cyclists' and pedestrians' current infrastructure usage. From the simulated MSS routes for 2020, we can directly identify on a trip segment what road type is used. For example, one can see when a bike trip uses paths specifically designed for cycling or whether the trip shares 
the road carriageways (i.e., state road, road, or street). Figure 10 indicates the average percentage and distance traveled on each road type per mode. As we can see, driving trips use the three appropriate road types: state road, road, and street. Cycling trips are routed using both roads, cycleways, and, to a minor degree, footways. On average, $13 \%$ of cycling trips use cycleways (with an average length of $755 \mathrm{~m}$ ), while nearly $80 \%$ use road carriageways (with an average length of $3 \mathrm{~km}$ ). Finally, walking trips use footways but mostly use the drivable network for routing (i.e., streets), which is due to the coding of the pedestrian networks that are almost nonexistent for many cities; for instance, pedestrian route planners (such as Google Maps) often resort to streets centerlines. Taking a closer look at cycling trips, Figure 11 shows the distribution of trips per percentage of cycleway usage. As expected, all driving trips use no cycleways. Remarkably, approximately $55 \%$ of cycling trips use none to low $(<1 \%)$ cycleways and only around $0.5 \%$ of trips use cycleways for the vast majority of their trip $(>80 \%)$. This questions the cycling network's efficiency because most cycling trips do not ride on existing dedicated cycling infrastructure.

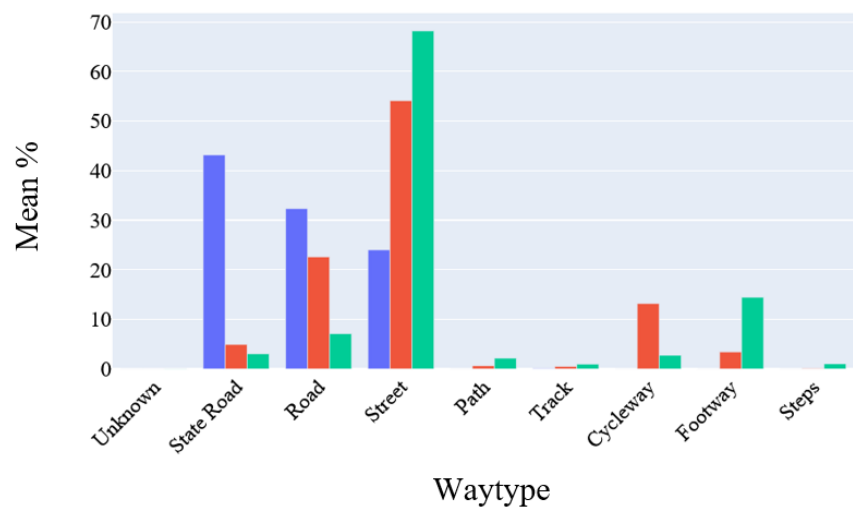

(a) Mean percentage of waytype used per mode.

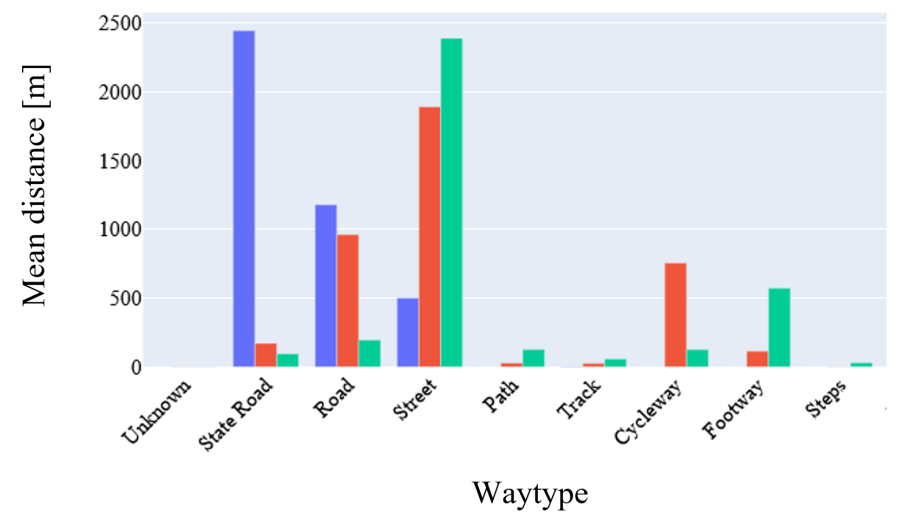

(b) Mean distance of waytype used per mode.

$$
\text { Mode } \rightarrow \text { driving } \rightarrow \text { cycling } \rightarrow \text { walking }
$$

Figure 10. Different average road types (waytype) used per mode for all MSS 2020 trips.

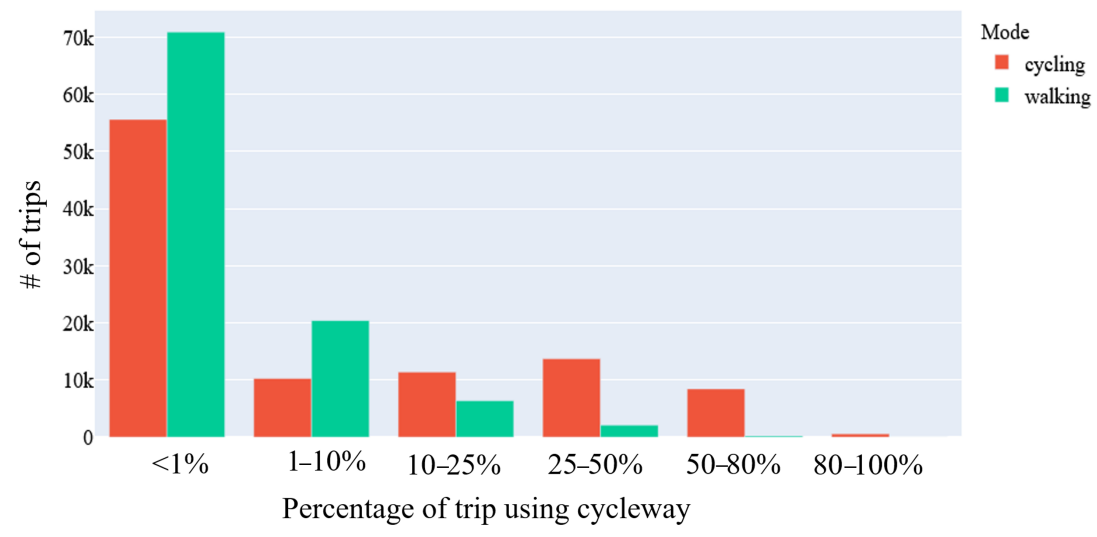

Figure 11. Percentage of cycleway used per trip for all modes using MSS in 2020.

These results are in line with other works, which also show that cycling trips are mostly performed in conjunction with road and cycleways. For example, Rupi and Schweizer [33] found that $26 \%$ of the cycling trips in Bologna are conducted in cycle lanes, and Hood et al. [34] estimate that $28 \%$ of trips in San Francisco was made in cycle lanes. These numbers contrast with our case, exhibiting a much lower average proportion of the trip being made in cycle lanes, which possibly indicates that the current dedicated cycling network is still insufficient. 


\subsection{Circuity Inequality}

Finally, we look at the Gini coefficient of Lisbon's parishes. Gini coefficient [35] can be used to measure the inequality of a given frequency distribution. While it is typically applied to identify income or wealth inequalities, it also identifies other types of inequalities. Here, we employ the Gini coefficient to see how disparate circuity is across our case study's three modes. Results close to zero indicate an equal distribution, while values similar to 1 indicate a highly unequal distribution. Our results indicate a Gini coefficient of 0.278 for driving, 0.223 for cycling, and 0.120 for walking trips, suggesting that circuity for walking is more equitable over the city than cycling and driving, in which circuity levels are more uneven across the city.

Figure 12 further shows Gini indexes across Lisbon's parishes. Figure 13 represents the MSS distribution of cycling circuity values for 2020 . About $84 \%$ of trips' circuity is below 2, $50 \%$ below 1.5 , and only around $1 \%$ is below 1.1 . Thus, in $50 \%$ of bike trips, cyclists have to cycle $50 \%$ more than a direct route, and only a marginal number of trips are somewhat direct (i.e., circuity close to 1 ). These results highlight that equality across circuity is very distinct between Lisbon's parishes for both cycling and driving. Walking appears to be more equal across Lisbon. This inequality analysis can be used to identify city areas that have low accessibility for a certain mode when compared to other areas in the city.

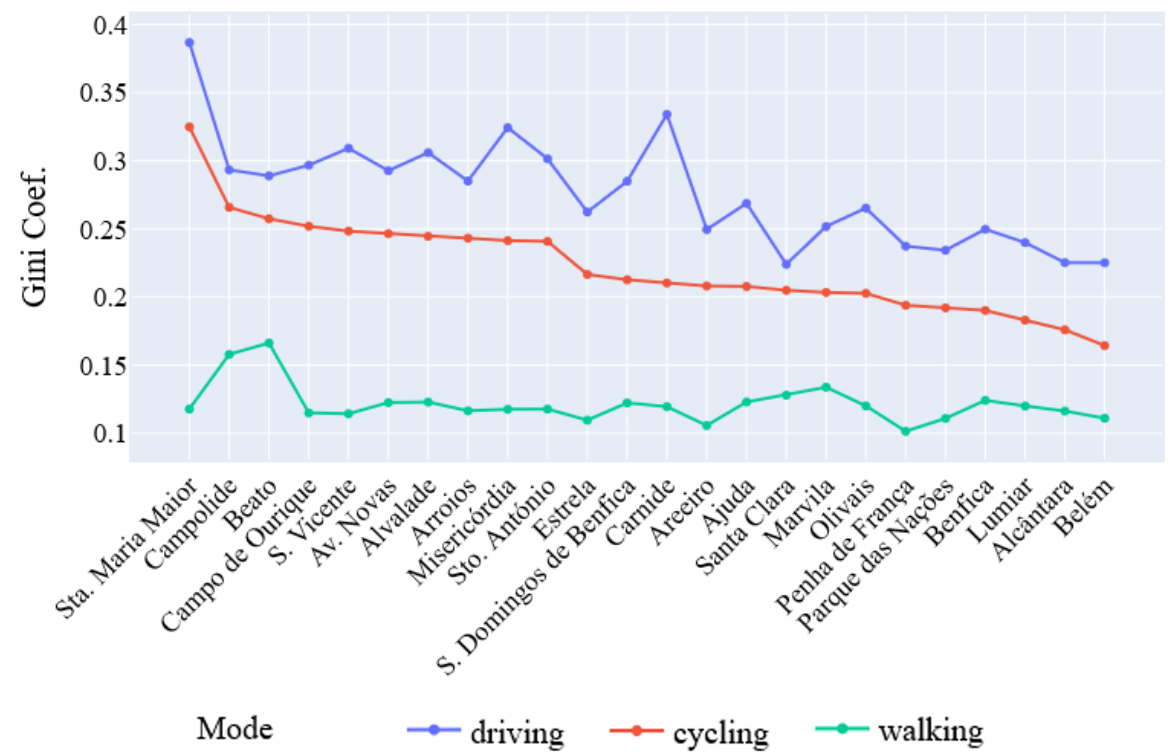

Figure 12. Gini coefficient for Lisbon's parishes in 2020.

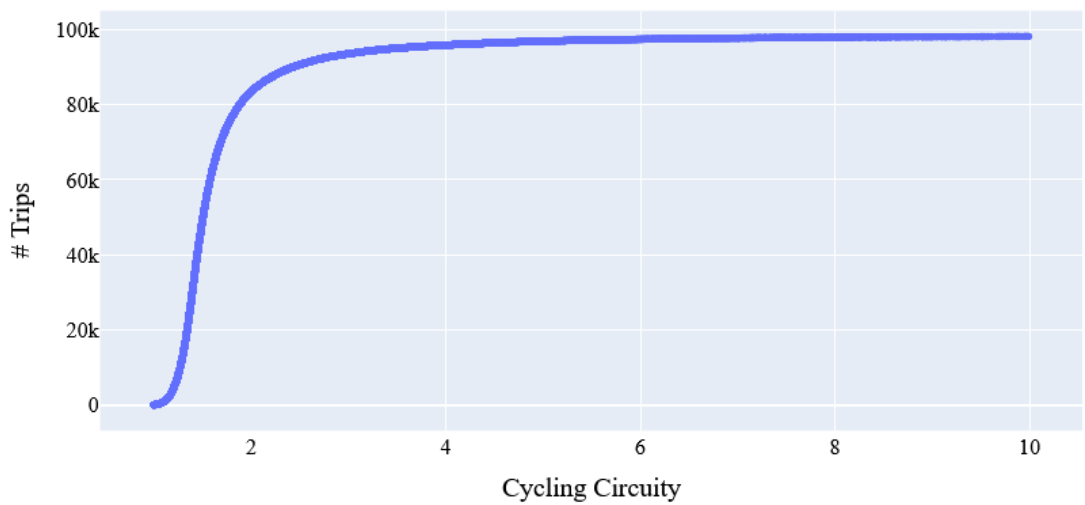

Figure 13. Distribution of cycling circuity values for all MSS cycling trips in 2020. 


\section{Discussion}

Circuity serves as a metric to measure trip routes' attractiveness and is suited to analyze and compare cities and city regions. Our research uses circuity to compare two circuity computation approaches to analyze Lisbon and its regions. Our results show that circuity is decreasing, especially for cycling and walking, consistent with changes that occurred in Lisbon during the analyzed period. We highlight that circuity can have very different results using two distinct computation approaches. For one, circuity analyzed for a demand-isotropic network of the city is recognizably different from the network's circuity used by existing demand (i.e., anisotropic). Thus, when using circuity as a measure of planning, the definition of the objective must be clear: either to improve the city as a whole by searching unraveled potential to satisfy demand or to prioritize the network used de facto.

Lisbon's parishes showed different circuity trends. Some parishes had similar circuity patterns for driving, cycling, and walking, while others varied substantially among modes. As a whole, our results support Cubukcu [6]'s and Yen et al. [19]'s ideas that circuity must be analyzed at lower scales rather than at the city scale. Different areas of the city generated considerably different results. Categorizing trips into different lengths also enabled a more refined analysis. We noticed that as trip distance increases, circuity tends to decrease. Furthermore, comparing the two sampling approaches yields key differences on the simulated trips when split in trip distances. RS yields more medium-length distances (approximately $76 \%$ of all trips between 2 and $8 \mathrm{~km}$ ) than the demand-driven MSS (where $2-8 \mathrm{~km}$ trips represent about $48 \%$ of all trips). This means that analyzing the average circuity of all trips versus categorized distance trips could misrepresent the actual circuity of trips and the analysis of urban networks. However, the MSS approach analyzes the origin-destination demand of citizens over the urban network, and while the simulated routes may not correspond to the true routes chosen by citizens, it allows for a general analysis of the topology of urban networks. Such analysis can be used to quickly analyze the impact of different possible interventions on the urban space. For trips from 2 to $8 \mathrm{~km}$ (which include the average trip distance in Lisbon [26]), we noticed that driving circuity is, in some cases, lower than cycling and walking circuities. Thus, being the favorite mode by Lisbonians, private cars can be seen as the most attractive when it comes to distance traveled. If the municipality's current goal is to promote more sustainable modes by replacing private motorized modes [36], then a clear emphasis must be placed on increasing such sustainable modes' attractiveness.

Looking at how circuity evolved over the years for Lisbon's parishes, we also note interesting evidence of how circuity has changed for cycling trips. As shown, cycling circuity has changed considerably alongside many improvements and Lisbon's cycling network extension. Notably, we highlight how circuity has changed for cycling trips and the impact that such infrastructure has had on individuals' trips. However, in some cases, such infrastructures' construction shows no apparent impact on circuity levels, suggesting that they are either not attractive in terms of distance traveled or that they are not appropriate for the trip. Another interesting fact relates to the length of existing cycle lanes, and their usage for trips inside the same parish. For example, cycling trips inside Avenidas Novas are $16.31 \%$ made in cycle lanes, with $16.65 \mathrm{~km}$ of existing cycles lane in Avenidas Novas. In Beato, which has only $1.26 \mathrm{~km}$ of cycle lanes, $23.72 \%$ of bicycle trips use cycle lanes. We hypothesize that Av. Novas' infrastructure is pervasive and is the backbone of Lisbon's cycling network, while it is not used for intra-parish trips, serving more to connect to other parts of the city. Meanwhile, in Beato's case, the small yet meaningful infrastructure is vital for accommodating cycling local (intra-parish) trips. Such inspection allows for a more generalized analysis of any cities street networks to understand what infrastructures are being used, and by which user.

Looking at Lisbon's circuity inequalities, we notice that circuity is more uneven for driving than cycling, and cycling is more uneven than walking. These results suggest that while walking circuity is more or less equal throughout the city, cycling, and driving 
circuities seem to vary significantly across Lisbon. Such results suggest that a more detailed analysis can be made to locate inequalities. Such understanding can better pinpoint any city's location where changes ought to be made such that these spots do not suffer from being less accessible by a particular transport mode.

Finally, by analyzing road type allocation to trip routes, we can identify some other intriguing facts. Over half (55\%) of the cycling trips use no cycling infrastructure, which is a crucial takeaway, since cycling uptake is dependent on adequate infrastructures [31]. Lisbon's municipality has shown serious commitment and effort in creating and expanding its cycling network, yet it seems that this network is still far from providing all citizens with a safe and comfortable environment to cycle through. Another significant upshot was the number of footways used when walking. Only $18.22 \%$ of walking trips are made on walkways, equating to an average of $571 \mathrm{~m}$, while the vast majority of walking uses streets $(66.82 \%)$, which include residential streets or living streets. In Lisbon, almost all urban streets have sidewalks on one or both sides of the road. A finer analysis of OpenStreetMaps evidenced that only a small fraction of Lisbon's streets contain information regarding sidewalks' existence. Although assumptions can be made that pedestrians use sidewalks of the streets, they were not routed through footways, which implies a lack of information on Lisbon's existing walking network.

This study presented a methodology capable of efficiently computing and comparing circuity values for different transportation modes and different scale levels. Nevertheless, while on the one hand, it is based on open and volunteered geographic data, on the other hand, it is vastly based on the quality of such data. Our analysis of the data and results achieved highlight this. For example, walking trips would not use walkways, which points out the lack and quality of pedestrian infrastructure data. In Lisbon, the vast majority of streets have sidewalks, and the impact of such defective representation of the pedestrian network is not relevant. However, it may not be the case for other cities where similar analyses are made.

Additionally, one of our sampling techniques is based on origin-destination points that were collected in 2017. However, these OD pairs may change over time, depending on attracting or deterring factors, locations, and purposes of trips. IMob [26] is the only survey in Lisbon that captured such mobility patterns and trends. Arguments may question the validity of such use of points over time, yet our goal was to assess the evolution and transformation of circuity over time, which was impossible without using such data in a more general timeframe.

Finally, we simulate trips based on the preferred route and not for the shortest distance trip. This choice in modeling reflects trips that people tend to make and may not always correspond to the shortest possible path. Calculating and comparing circuities over the shortest trips may have different results, possibly underscoring even more disparate results, leading to bigger differences per mode and distance traveled.

\section{Conclusions}

Circuity serves as a metric to measure the attractiveness of trip routes. It can be used to analyze and compare regions, cities, or even city districts. In this work, we use circuity to compare driving, cycling, and walking trips in Lisbon from 2013 to 2020. We use data from OpenStreetMaps and present a methodology that can be easily reproducible in other cities to simulate routes using different transport modes. We test two strategies to simulate routes and conclude that RS is appropriate to analyze the whole urban street network, considering a demand-isotropic network, while MSS should be used when there is a need to understand the network that citizens use.

Our results note that circuity decreases over time and identify many cases where this decrease is consistent with Lisbon's synchronous infrastructure changes. We compare Lisbon's parishes and identify several differences between them: how much inequality there is between the three sampled modes, how circuity changes for different trips' distances, and what road types are used for each model. Using our analysis, we can identify signifi- 
cant differences between driving, cycling, and walking. We postulate that this analysis can then target planning opportunities or identify locations that need changes. Thus, circuity serves as a valuable objective metric to compare modes and networks.

For future work, we identify avenues that ought to require more investigation. First, a particular emphasis has been put on the analysis of Lisbon's cycling circuity. Lisbon, a cycling starter city, should be compared to other cities whose focus and modal share are distinct, such as Copenhagen or Amsterdam, to see whether significant differences can be found between the two cities' typologies. This study can be used to create a benchmark of circuity, possibly identifying good and bad examples of circuity values and identify feasible circuity levels that can be achieved. Second, an optimization tool could be developed to identify which streets' infrastructures could change to achieve specific circuity and accessibility goals. While such a tool can improve circuity for a given mode, the impair or harm circuity for other modes may also lead to behavior changes and mode choices. Finally, public transportation and multimodal trips should also be considered.

Although this work only focused on three transport modes, public transportation should serve as a backbone to any city seeking sustainable mobility, and a circuity analysis should consider this. Additionally, trips using multiple modes should also be analyzed, as this mode choice is also significant and often the solution for better accessibility and mobility.

Author Contributions: Conceptualization, Miguel Costa, Manuel Marques, and Filipe Moura; methodology, Miguel Costa, Manuel Marques, and Filipe Moura; software, Miguel Costa; validation, Miguel Costa, Manuel Marques, and Filipe Moura; formal analysis, Miguel Costa, Manuel Marques, and Filipe Moura; investigation, Miguel Costa, Manuel Marques, and Filipe Moura; resources, Miguel Costa, Manuel Marques, and Filipe Moura; data curation, Miguel Costa; writing_original draft preparation, Miguel Costa, Manuel Marques, and Filipe Moura; writing—review and editing, Miguel Costa, Manuel Marques, and Filipe Moura; visualization, Miguel Costa; supervision, Manuel Marques and Filipe Moura. All authors have read and agreed to the published version of the manuscript.

Funding: This work was partially funded by Fundação para a Ciência e Tecnologia via grant [PD/BD/142948/2018], and LARSyS-FCT Project [UIDB/50009/2020].

Data Availability Statement: Code and materials are available at https:/ / github.com/U-Shift/cir cuity-lisbon, accessed on 28 June 2021.

Conflicts of Interest: The authors declare no conflict of interest.

\section{References}

1. Marshall, S. Streets and Patterns; Routledge: London, UK, 2004.

2. Winters, M.; Brauer, M.; Setton, E.M.; Teschke, K. Built environment influences on healthy transportation choices: Bicycling versus driving. J. Urban Health 2010, 87, 969-993. [CrossRef]

3. Broach, J.; Dill, J.; Gliebe, J. Where do cyclists ride? A route choice model developed with revealed preference GPS data. Transp. Res. Part A Policy Pract. 2012, 46, 1730-1740. [CrossRef]

4. Jang, S.; An, Y.; Yi, C.; Lee, S. Assessing the spatial equity of Seoul's public transportation using the Gini coefficient based on its accessibility. Int. J. Urban Sci. 2017, 21, 91-107. [CrossRef]

5. Huang, J.; Levinson, D.M. Circuity in urban transit networks. J. Transp. Geogr. 2015, 48, 145-153. [CrossRef]

6. Cubukcu, K.M. Using circuity as a network efficiency measure: The example of Paris. Spat. Inf. Res. 2020, 29, 163-172. [CrossRef]

7. Derrible, S.; Kennedy, C. Characterizing metro networks: State, form, and structure. Transportation 2010, 37, 275-297. [CrossRef]

8. Parthasarathi, P. Network structure and metropolitan mobility. J. Transp. Land Use 2014, 7, 153-170. [CrossRef]

9. Ahmadzai, F.; Rao, K.L.; Ulfat, S. Assessment and modelling of urban road networks using Integrated Graph of Natural Road Network (a GIS-based approach). J. Urban Manag. 2019, 8, 109-125. [CrossRef]

10. Levinson, D. Network structure and city size. PLoS ONE 2012, 7, e29721.

11. Crucitti, P.; Latora, V.; Porta, S. Centrality in networks of urban streets. Chaos Interdiscip. J. Nonlinear Sci. 2006, 16, 015113. [CrossRef] [PubMed]

12. Boeing, G. Urban spatial order: Street network orientation, configuration, and entropy. Appl. Netw. Sci. 2019, 4, 67. [CrossRef]

13. Carpio-Pinedo, J.; Martínez-Conde, J.A.; Daudén, F.L. Mobility and Urban Planning Integration at City-regional Level in the Design of Urban Transport Interchanges (EC FP7 NODES Project-Task 3.2.1.). Procedia Soc. Behav. Sci. 2014, 160, $224-233$. [CrossRef]

14. Dingil, A.E.; Rupi, F.; Stasiskiene, Z. A macroscopic analysis of transport networks: The influence of network design on urban transportation performance. Int. J. Transp. Dev. Integr. 2019, 3, 331-343. [CrossRef] 
15. Levinson, D.; El-Geneidy, A. Network Circuity and the Location of Home and Work. In Proceedings of the University Transport Study Group Conference, Harrowgate, UK, 3-5 January 2007.

16. Levinson, D.; El-Geneidy, A. The minimum circuity frontier and the journey to work. Reg. Sci. Urban Econ. 2009, 39, 732-738. [CrossRef]

17. Giacomin, D.J.; Levinson, D.M. Road network circuity in metropolitan areas. Environ. Plan. B Plan. Des. 2015, 42, 1040-1053. [CrossRef]

18. Boeing, G. The morphology and circuity of walkable and drivable street networks. In The Mathematics of Urban Morphology; Springer: Cham, Switzeland, 2019; pp. 271-287.

19. Yen, Y.; Zhao, P.; Sohail, M.T. The morphology and circuity of walkable, bikeable, and drivable street networks in Phnom Penh, Cambodia. Environ. Plan. Urban Anal. City Sci. 2021, 48, 169-185. [CrossRef]

20. O'Sullivan, S.; Morrall, J. Walking distances to and from light-rail transit stations. Transp. Res. Rec. 1996, 1538, 19-26. [CrossRef]

21. Dill, J. Measuring network connectivity for bicycling and walking. In Proceedings of the 83rd Annual Meeting of the Transportation Research Board, Washington, DC, USA, 11-15 January 2004; pp. 11-15.

22. Barrington-Leigh, C.; Millard-Ball, A. The world's user-generated road map is more than $80 \%$ complete. PLoS ONE 2017, 12, e0180698. [CrossRef]

23. Heidelberg Institute for Geoinformation Technology (HeiGIT). OpenRouSteService. 2020. Available online: https://github.com / GIScience/openrouteservice (accessed on 1 September 2020).

24. Krizek, K.J. Two approaches to valuing some of bicycle facilities' presumed benefits: Propose a session for the 2007 national planning conference in the city of brotherly love. J. Am. Plan. Assoc. 2006, 72, 309-320. [CrossRef]

25. Rupi, F.; Poliziani, C.; Schweizer, J. Data-driven bicycle network analysis based on traditional counting methods and GPS traces from smartphone. ISPRS Int. J. Geo-Inf. 2019, 8, 322. [CrossRef]

26. INE, I. P. Mobilidade e Funcionalidade do TerritóRio nas Áreas Metropolitanas do Porto e de Lisboa 2017; Instituto Nacional de Estatística, I. P.: Lisboa, Portugal, 2018.

27. Félix, R. Bicycle Mobility Management-User's Needs and Preferences for Network Planning and Management in the City of Lisbon. Master's Thesis, Instituto Superior Técnico, Universidade Técnica de Lisboa, Lisboa, Portugal, 2012.

28. Moura, F.; Silva, J.; Picado Santos, L. Growing from incipient to potentially large cycle networks: Screening the road network of the consolidated urban area of Lisbon. Eur. J. Transp. Infrastruct. Res. 2017, 17, 170-190.

29. Sequera, J.; Nofre, J. Touristification, transnational gentrification and urban change in Lisbon: The neighbourhood of Alfama. Urban Stud. 2020, 57, 3169-3189. [CrossRef]

30. INE, I. P. Censos 2011 Resultados Definitivos-Portugal; Instituto Nacional de Estatística, I. P.: Lisboa, Portugal, 2012.

31. Félix, R.; Cambra, P.; Moura, F. Build it and give 'em bikes, and they will come: The effects of cycling infrastructure and bike-sharing system in Lisbon. Case Stud. Transp. Policy 2020, 8, 672-682. [CrossRef]

32. GISLisboa. Rede Ciclável. 2020. Available online: https://geodados-cml.hub.arcgis.com/datasets/440b7424a6284e0b9bf11179b 95bf8d1_0 (accessed on 15 December 2020).

33. Rupi, F.; Schweizer, J. Evaluating cyclist patterns using GPS data from smartphones. IET Intell. Transp. Syst. 2018, 12, 279-285. [CrossRef]

34. Hood, J.; Sall, E.; Charlton, B. A GPS-based bicycle route choice model for San Francisco, California. Transp. Lett. 2011, 3, 63-75. [CrossRef]

35. Gini, C. Variabilità e Mutabilità: Contributo allo Studio delle Distribuzioni e delle Relazioni Statistiche; C. Cuppini: Bologna, Italy, 1912.

36. de Lisboa, C.M. MOVE LISBOA Visão Estratégica para a Mobilidade 2030; Technical Report; Câmara Municipal de Lisboa: Lisboa, 2020. 